\title{
OR10-005 - Treatment responses in TRAPS: Eurofever/ Eurotraps
}

\author{
HJ Lachmann*, K Minden, L Obici, A Naselli, A Insalaco, V Hentgen, R Merino, C Modesto, N Toplak, R Berendes, \\ R Cimaz, A Jansson, A Martini, P Woo, I Touitou, M Gattorno \\ From 7th Congress of International Society of Systemic Auto-Inflammatory Diseases (ISSAID) \\ Lausanne, Switerland. 22-26 May 2013
}

\section{Introduction}

TRAPS is a rare lifelong disease. Optimal treatment is not established but tends to rely either on corticosteroids with risks of known short and long term side effects or anti cytokine agents which are expensive, and both types of agents lead to higher risk of infection.

\section{Objectives}

To analyze treatments used and their responses in patients with clinical TRAPS associated with a pathogenic sequence variants (PSV) in TNFRSF1A enrolled in the Eurofever/Eurotraps registry.

\section{Methods}

The Eurofever Project (agreement n 2007332, EAHC) built a common web-based registry for all Autoinflammatory diseases in collaboration with the Eurotraps Project (FP7, HEALTH-F2-2008-200923).

\section{Results}

In total there was treatment data on 113 patients with 45 different PSV of TNFRSF1A. Patients came from 14 countries and 94.5\% were of European Caucasian ancestry. 16 patients had only received symptomatic treatment.

Of 48 patients given steroids only with attacks 20 $(42 \%)$ reported complete success (CR) in terminating acute attacks but 38 (79\%) were either converted to biologic therapy or had them added to improve disease control. Of 22 patients on maintenance steroids $6(27 \%)$ reported complete attack prevention but 14 (64\%) were converted to biologic therapy. 37 patients received etanercept (in the 19 where data was available for a median of 51 months). 9 patients had a CR and 26 a partial response
(PR). 10 remain on etanercept. Of the 27 who discontinued etanercept inadequate disease response was sole or contributory reason for discontinuing etanercept in 21 and side effects in 9.20 patients converted to anti IL-1 therapy. 38 patients received anakinra. 34 (89\%) reported a CR and 4 a PR. $92 \%$ remain on anakinra with a median treatment duration date of 23 months (range 1- 89 months).

\section{Conclusion}

This is the largest survey of treatment of TRAPS to date. The marked predominance of patients from Western Europe may be reflected in the high use of biologic agents which are not necessarily widely available. The most significant findings are that corticosteroids are effective in more than $40 \%$ of patients initially but almost $80 \%$ of patients have been converted to anti cytokine agents. Anakinra is completely effective in $89 \%$ of cases and continued as long-term treatment in $92 \%$. Its use is associated with a $90 \%$ reduction in the requirement for corticosteroids to treat acute attacks. Etanercept is significantly less effective and is discontinued in almost $75 \%$ of cases. Although these data strongly support use of anti IL-1 agents to treat TRAPS follow up remains short and reevaluation will be required.

\section{Competing interests}

None declared.

Published: 8 November 2013

doi:10.1186/1546-0096-11-S1-A188

Cite this article as: Lachmann et al:: OR10-005 - Treatment responses in TRAPS: Eurofever/ Eurotraps. Pediatric Rheumatology 2013 11(Suppl 1): A188.

for PRINTO, Eurotraps/Eurofever Projects, Genoa, Italy

(C) 2013 Lachmann et al; licensee BioMed Central Ltd. This is an Open Access article distributed under the terms of the Creative 